\title{
Acceso a la educación superior en Chile desde la financiación privada: entre la inequidad estructural y la oportunidad individual
}

\author{
Pablo Rivera-Vargas \\ Universitat de Barcelona. España. \\ Universidad Andrés Bello. Chile. \\ pablorivera@ub.edu \\ Victor Climent Sanjuan \\ Universitat de Barcelona. España. \\ vcliment@ub.edu
}

\section{René Rivera Bilbao}

Universidad de las Artes y las Ciencias de la Comunicación. Chile. rene.rivera@uniacc.edu

Recibido: 30/3/2020

Aceptado: 22/7/2020

Publicado: 5/7/2021

\section{Resumen}

El principal indicador para evaluar la equidad del sistema de educación superior en Chile ha sido la ampliación de la matrícula. Entre las medidas adoptadas para su consecución se encuentra el uso del Crédito con Aval del Estado (CAE) como herramienta para financiar los estudios universitarios. El objetivo de esta investigación ha sido analizar el impacto de dicha política, tanto en la configuración del mercado universitario actual como en la percepción de sus «beneficiarios». A través de un enfoque metodológico mixto, que incluyó revisión documental y entrevistas individuales a estudiantes beneficiarios del CAE, se generaron tres dimensiones de análisis. Los resultados evidencian la existencia de interfaces complejas entre los resultados macroeconómicos y la experiencia de los estudiantes. En efecto, mientras una mirada a la objetividad del sistema demuestra el soporte neoliberal y el refuerzo que supone a la «bancarización» del financiamiento educativo, la perspectiva de los beneficiarios es que el CAE representaría una oportunidad única de acceso a la educación superior.

Palabras clave: acceso a la educación; universidad; administración de la educación; financiación privada

Resum. Accés a l'educació superior a Xile des del finançament privat: entre la iniquitat estructural $i$ l'oportunitat individual

El principal indicador per avaluar l'equitat del sistema d'educació superior a Xile ha estat l'ampliació de la matrícula. Entre les mesures adoptades per aconseguir-ho es troba l'ús del Crèdit amb Aval de l'Estat (CAE) com a eina per finançar els estudis universitaris. L'objectiu d'aquesta investigació és analitzar l'impacte de l'esmentada política, tant en la configuració del mercat universitari actual com en la percepció dels seus «beneficiaris». A través d'un enfocament metodològic mixt, que va incloure revisió documental i entrevistes individuals a estudiants beneficiaris del CAE, es van generar tres dimensions d'anàlisi. Els 
resultats evidencien l'existència d'interfícies complexes entre els resultats macroeconòmics $\mathrm{i}$ l'experiència dels estudiants. En efecte, mentre una mirada a l'objectivitat del sistema demostra el suport neoliberal i el reforç que suposa a la «bancarització» del finançament educatiu, la perspectiva dels beneficiaris és que el CAE representaria una oportunitat única d'accés a l'educació superior.

Paraules clau: accés a l'educació; universitat; administració de l'educació; finançament privat

\section{Abstract. Access to higher education in Chile through private financing: Between structural inequality and individual opportunity}

The main indicator for evaluating the equity of the higher education system in Chile has been the expansion of enrollment. Among the measures adopted to achieve such equity is the creation of a State Guaranteed Student Credit (SCSC) as a tool to finance university studies. The objective of this study is to analyze the impact of this policy, both in the configuration of the current university market and in the perception of its "beneficiaries." Through a mixed methodological approach, which included documentary review and individual interviews with students who were beneficiaries of the SCSC, three dimensions of analysis were generated. The results show the existence of complex interfaces between the educational reality, public policy, and the experience of students. In effect, while a look at the objectivity of the system demonstrates the neoliberal support and reinforcement of the "bankarization" of educational financing, the perspective of the beneficiaries is that the SCSC represents a unique opportunity to access higher education.

Keywords: access to education; university; administration of education; private financing

\section{Sumario}

1. Introducción

2. Una aproximación a la historia reciente del financiamiento universitario en Chile: desde la cobertura hasta el lucro bancario
3. Marco metodológico

4. Resultados

5. Discusión y conclusiones

Referencias bibliográficas

\section{Introducción}

El acceso a la educación superior ha sido un desafío sustantivo en el diseño de políticas públicas de mayor equidad social. En Chile, una de las principales propuestas al respecto ha sido la creación del Crédito con Aval del Estado (CAE) $)^{1}$. Se trata de una medida implementada por el gobierno de Ricardo Lagos $^{2}$ bajo el argumento de que el Estado, en un contexto de restricción presupuestaria, requiere del soporte de la banca privada para asegurar el acce-

1. Es un préstamo para cursar estudios de educación superior establecido mediante la ley n. ${ }^{\circ} 20.027$, aprobada por el Congreso Nacional de Chile.

2. Presidente de Chile en el periodo 2004-2010. 
so y la financiación de la educación superior, particularmente para aquellos sectores sociales que, cumpliendo con los requisitos académicos de acceso, no pueden costear sus estudios universitarios (Larraín y Zurita, 2008). El CAE es un sistema basado en la entrega de un crédito por parte del sistema financiero bancario a las instituciones de educación superior, cuyo retorno, por parte de los estudiantes beneficiarios, está avalado o asegurado por los recursos públicos del Estado (Rivera, Climent, Espinoza y Rivera-Vargas, 2018). De esta manera, el CAE institucionaliza una relación donde el Estado se erige como el aval de estos ante la banca (Dooner y Mena, 2018).

La investigación marco que da origen al presente artículo tuvo como objetivo analizar el funcionamiento del CAE a partir de los conceptos de equidad de acceso a la educación superior y de movilidad social en Chile. Con este fin, además de consultar y analizar datos oficiales y resultados de informes relevantes en la materia, se entrevistó a estudiantes que financiaban sus estudios mediante el CAE y que, además, pertenecían a las universidades con el porcentaje más alto de alumnos de este grupo.

El presente artículo profundiza en una de las dimensiones principales de la investigación, que tiene que ver con la coherencia y las interfaces entre la información racional sobre el aumento del acceso a la universidad gracias al CAE, el endeudamiento del estudiantado y la percepción de este sobre dicho instrumento como un beneficio que favorezca su propia movilidad social. Ante esto, hemos planteado el siguiente interrogante guía: ¿Cuáles son las principales coherencias e interfaces entre los resultados del CAE en tanto instrumento de acceso a la educación superior en Chile y la percepción del estudiantado beneficiario?

Con el fin de dar respuesta a esta pregunta, el artículo se ha organizado en los siguientes cuatro apartados: «Una aproximación a la historia reciente del financiamiento universitario en Chile», «Marco metodológico», «Resultados» $\mathrm{y}$ «Discusión y conclusiones».

\section{Una aproximación a la historia reciente del financiamiento universitario en Chile: desde la cobertura hasta el lucro bancario}

La génesis del CAE está vinculada estrechamente con los principios y las lógicas del modelo de desarrollo social y económico impuesto en Chile durante la dictadura cívico-militar de Augusto Pinochet (1973-1990). En 1981, y con el objetivo de satisfacer la creciente demanda de matrículas en educación superior, el Estado permitió la creación de numerosas instituciones universitarias de capital privado, con la condición de que no tuvieran ánimo de lucro (Bernasconi y Rojas, 2004; Sisto, 2013). Para facilitar la implementación de este nuevo modelo se entregó a la banca la prerrogativa de financiar la educación superior de miles de estudiantes con especiales dificultades económicas, a partir de la concesión de créditos que contarían con el aval del Estado en el supuesto del impago de la deuda, con tasas promedio de un $60 \%$ anual (Banco Mundial, 2011). 
Una vez recuperada la democracia en Chile en el año 1990, los gobiernos del pacto político denominado Concertación ${ }^{3}$ establecieron una serie de políticas públicas con el objetivo de expandir el acceso a la educación superior y que esta pudiera ser accesible a una base social mucho más amplia (Rivera y Paredes, 2018; Larraín y Zurita, 2008; Atria, 2017; Rivera-Polo, Rivera-Vargas y AlonsoCano, 2018). Hasta comienzos del año 2005 la alternativa más común para los estudiantes económicamente más vulnerables consistía en solicitar el Fondo Solidario de Crédito Universitario, que exclusivamente beneficiaba a los estudiantes con buenos resultados en la Prueba de Selección Universitaria que deseaban financiar su educación en una de las veinticinco universidades del Consejo de Rectores (CRUCH) ${ }^{4}$. La nueva modalidad crediticia del CAE (Ley $20.027)^{5}$ fue promulgada el 1 de junio de 2005 y pasó a otorgar dicho beneficio a miles de jóvenes que necesitaban apoyo económico sin que importara si estudiaban en una institución pública o privada, o que esta formara parte, o no, del Consejo de Rectores. De hecho, esta medida tuvo un gran impacto social, puesto que desde 2006 hasta 2011 más 360 mil estudiantes sufragaron sus estudios superiores a través del CAE (Salazar y Leihy, 2013).

Inicialmente la tasa de interés del CAE era de un 5,8\%, mientras que la del Fondo Solidario de Crédito Universitario se situaba en un 2\%. Esta diferencia porcentual y, por ende, el alto endeudamiento de los estudiantes con el $\mathrm{CAE}$, fue uno de los detonantes que justamente impulsó la gran movilización estudiantil y social en Chile del año 2011 e implicó la paralización de las universidades durante un periodo aproximado de seis meses (Mayol y Azócar, 2011; Sisto, 2013; Sandoval y Carvallo, 2019).

En pos de resolver el conflicto, el entonces ministro de Educación de Chile, Felipe Bulnes, anunció el 17 de agosto del 2011 que el Gobierno asumiría el costo fiscal de 100 mil millones de pesos anuales ${ }^{6}$, lo que supondría bajar la tasa de interés del CAE de un 5,8\% a un 2\% (Sisto, 2013). Además, se crearía una agencia estatal que se haría cargo, a partir de marzo del año 2013, del sistema de financiamiento de los estudiantes de educación superior.

Dicha ley finalmente fue promulgada el año 2012, pero su implantación no ha estado exenta de numerosos problemas. Por un lado, el movimiento estudiantil siguió con sus demandas de una educación superior pública y gratuita, tensando la noción neoliberal, tanto de calidad (Colella y Díaz-Salazar, 2016; Giroux, Rivera-Vargas y Passerón, 2020) como de universidad (JiménezPeralta, 2019) que predominaba, lo que nuevamente trabó el diálogo con el Gobierno. Y, por otro lado, se constató que una parte importante de los estudiantes y exestudiantes de educación superior no cumplían alguno de los requisitos exigidos para acceder a los beneficios de la nueva regulación (Rivera et

3. Coalición de centroizquierda que gobernó Chile desde el 11 de marzo de 1990 hasta el 11 de marzo de 2010.

4. Organización de universidades estatales y privadas tradicionales chilenas fundadas antes de 1981 o derivadas de ellas. Más información: <http://www.consejoderectores.cl/universidades>.

5. Más información: <https://www.leychile.cl/Navegar?idNorma=239034>.

6. Equivalentes a 135 millones de euros aproximadamente (12/03/2020). 
al., 2018) ${ }^{7}$. Además, otro grupo importante también optó por no acogerse a esta negociación, ya fuera por haber finalizado sus estudios o por estar próximo a hacerlo (Banco Mundial, 2011; Sisto, 2013). Básicamente, el principal problema que se presentó tuvo que ver con las altas tasas de intereses de sus deudas y con la constatación de que la mayoría de los alumnos, una vez finalizados sus estudios, se veían obligados a destinar más del $30 \%$ de sus ingresos mensuales a pagar la deuda del CAE (Ingresa, 2017).

En este contexto, el debate en Chile en torno a la política de financiamiento a través del CAE sigue sin resolverse. Por un lado, hay quienes resaltan el potencial del CAE como una iniciativa que, siendo perfectible, sí ha podido favorecer el acceso a la educación superior de los estudiantes provenientes de los sectores más vulnerables de la población chilena (Rodríguez-Ponce, 2012; Brunner, 2015; Miranda-Hiriart, 2016). Por otro lado, hay quienes denuncian que la política de financiamiento de la educación superior a través del CAE representa principalmente una oportunidad para la banca, que se lucra gracias a las necesidades formativas de millones de estudiantes en Chile (Banco Mundial, 2011; Dooner y Mena, 2018; Inzunza, Assael, Cornejo y Redondo, 2019).

\section{Marco metodológico}

La investigación fue abordada a partir de un proceso de combinación de estrategias cuantitativas y cualitativas. En primer lugar, a nivel cuantitativo se llevó a cabo una revisión documental y de datos numéricos secundarios. Según lo planteado por Scribano y De Sena (2009) respecto a la fiabilidad y a la eficiencia del análisis documental y a la búsqueda de información secundaria relevante, se han identificado y analizado un conjunto de datos numéricos provenientes de diferentes informes y reportes, tanto de organismos públicos como de organizaciones e instituciones de reconocida legitimidad científica y académica en el ámbito del análisis de las políticas educativas y económicas en Chile. Los documentos seleccionados han sido los siguientes:

- Encuesta de Caracterización Socioeconómica Nacional (CASEN) ${ }^{8}$.

- Sistema de Información de Educación Superior en Chile, Ministerio de Educación (SIES)? .

- Cuenta pública comisión Ingresa ${ }^{10}$.

7. Haber recibido un crédito CORFO, tener deudas de más de dos meses con el sistema anterior del CAE o haber abandonado sus estudios.

8. En esta investigación se analizaron los datos correspondientes a la encuesta CASEN del año 2013. Más información: <http://encuestacasen.cl/>.

9. Sobre la base de registros individuales de estudiantes, titulados y académicos (entre otros) en Chile. En esta investigación se analizaron los datos existentes hasta el 31/12/2016. Más información: <http://www.mifuturo.cl/sies/>.

10. Servicio público de carácter descentralizado creado en 2005. Para esta investigación se analizaron los datos del año 2017. Más información: <https://bit.ly/2ZaiKBJ>. 
- Informe sobre Pasivos Contingentes (INPC) de la Dirección de Presupuestos del Gobierno de Chile (DIPRES) ${ }^{11}$.

En segundo lugar, en relación con la información cualitativa, se llevaron a cabo entrevistas activas individualizadas. Este instrumento es definido como una interacción en la cual los partícipes (el entrevistador o la entrevistadora y el entrevistado o la entrevistada) son asumidos como sujetos que interactúan abiertamente, aunque guiados temáticamente por una pauta flexible (Denzin, 2001; Holstein y Gubrium, 1995).

Las entrevistas activas fueron aplicadas a 20 estudiantes universitarios que han financiado sus estudios mediante el CAE en dos universidades privadas de Chile. Estas instituciones fueron seleccionadas por ser dos de las tres universidades que (respecto del total) contaban con el mayor porcentaje de estudiantes que financiaban sus estudios a través del CAE.

A partir de la propuesta de Valles (2007) y Fernández-Ferrer y Forés (2016) sobre los criterios de triangulación de análisis de datos en estudios mixtos, la información secundaria (de corte cuantitativa) y primaria (de corte cualitativa) generada, ha sido inicialmente analizada de forma independiente, y, posteriormente, agrupada en categorías comunes. Esta agrupación ha permitido que los resultados hayan sido organizados a partir de tres categorías de contenido, donde se han contrapuesto los antecedentes macroeconómicos con los discursos y las manifestaciones de los entrevistados (Scribano y De Sena, 2009). Este ejercicio ha permitido evidenciar coherencias, pero también interfaces entre las evidencias, lo que nos ha posibilitado construir una respuesta a la pregunta guía del presente artículo.

\section{Resultados}

En este apartado se presenta una parte significativa del desarrollo analítico de las categorías: «Favoreciendo el acceso a la educación superior», «¿Equidad en el acceso?» $\mathrm{y}$ «Lucro para la banca y oportunidad individual».

\subsection{Favoreciendo el acceso a la educación superior}

Como hemos mencionado en la primera parte de este artículo, el problema del acceso a la educación superior ha influenciado una parte importante del debate sobre la equidad del sistema de educación superior en Chile (Banco Mundial, 2011; Rodríguez-Ponce, 2012; Brunner, 2015; Miranda-Hiriart, 2016; Dooner y Mena, 2018; Inzunza, Assael, Cornejo y Redondo, 2019). En este ámbito, y desde una perspectiva esencialmente macroeconómica, la promesa del aumento del acceso a la educación superior se habría cumplido

11. Aporta antecedentes sobre los compromisos financieros fiscales de la población chilena. Para esta investigación se analizaron los datos del informe del año 2015. Más información: <http:// www.dipres.gob.cl/598/articles-143031_doc_pdf.pdf>. 
Tabla 1. Evolución del acceso a la educación superior en Chile (1980-2015)

\begin{tabular}{|c|c|c|c|c|c|c|}
\hline \multirow{2}{*}{$\begin{array}{c}\text { Tipo de } \\
\text { institución }\end{array}$} & \multicolumn{2}{|c|}{1980} & \multicolumn{2}{|c|}{2005} & \multicolumn{2}{|c|}{2015} \\
\hline & $\begin{array}{c}\text { Número } \\
\text { instituciones }\end{array}$ & Matrícula & $\begin{array}{c}\text { Numero } \\
\text { instituciones }\end{array}$ & Matrícula & $\begin{array}{c}\text { Número } \\
\text { instituciones }\end{array}$ & Matrícula \\
\hline $\begin{array}{l}\text { Universidades } \\
\text { estatales o con } \\
\text { subsidio estatal }\end{array}$ & 8 & 118.978 & 25 & 207.096 & 25 & 304.577 \\
\hline $\begin{array}{l}\text { Universidades } \\
\text { privadas nuevas }\end{array}$ & 0 & 0 & 40 & 299.640 & 35 & 341.391 \\
\hline $\begin{array}{l}\text { Total universi- } \\
\text { dades }\end{array}$ & 8 & 118.978 & 60 & 506.736 & 60 & 645.968 \\
\hline $\begin{array}{l}\text { Institutos profe- } \\
\text { sionales }\end{array}$ & 0 & 0 & 81 & 15.001 & 43 & 373.171 \\
\hline $\begin{array}{l}\text { Centros de for- } \\
\text { mación técnica }\end{array}$ & 0 & 0 & 161 & 50.390 & 54 & 146.515 \\
\hline $\begin{array}{l}\text { Total educación } \\
\text { superior }\end{array}$ & 8 & 118.978 & 302 & 622.127 & 157 & 1.165 .654 \\
\hline $\begin{array}{l}\text { Cobertura } \\
\text { (población de } \\
18 \text { a } 24 \text { años) }\end{array}$ & \multicolumn{2}{|c|}{$7,5 \%$} & \multicolumn{2}{|c|}{$11 \%$} & \multicolumn{2}{|c|}{$53,1 \%$} \\
\hline
\end{tabular}

Fuente: elaboración propia a partir de los datos oficiales del Ministerio de Educación (SIES, 2017).

ampliamente. Como podemos observar en la tabla 1, en 1980 (un año antes de que se pusieran en marcha las universidades privadas en Chile) se registró una matrícula total de 118.978 estudiantes. En 2005 (un año antes de que comenzara a operar el CAE) la cifra subió a 663.679 alumnos. Finalmente, en 2015 (dos años después de que se implementara una parte importante de los cambios generados por la gran movilización estudiantil del año 2011) se llegó a un total de 1.165 .654 matriculados.

Más allá del explícito cuestionamiento que hizo el movimiento estudiantil del año 2011 en referencia al hecho de que el destino de los fondos avalados por el Estado fuera mayoritariamente para instituciones (IES) de carácter privado (Sisto, 2013), en el gráfico 1 se puede apreciar cómo, desde el año 2010 hasta el 2015, el número de deudores del CAE continuó en un aumento sostenido.

Tal como se puede apreciar en la tabla 2, estos datos tienen su correlato en la percepción de los propios estudiantes, que reconocen el aumento de la cobertura en educación superior y que, más allá de los cuestionamientos sociales al CAE como herramienta de financiamiento de la educación superior, es valorado positivamente como oportunidad de acceso.

La posibilidad de acceso y financiamiento - independientemente del modo en que ambos se materialicen - se instalan como el horizonte valorativo de la subjetividad estudiantil. Para ellos el CAE representa prácticamente la única posibilidad de ingresar en la educación superior, y esta constatación opera como matriz interpretativa global que impele a considerar positivamente el financiamiento crediticio de la educación universitaria. 
Gráfico 1. Número de deudores del CAE

650.000

550.000

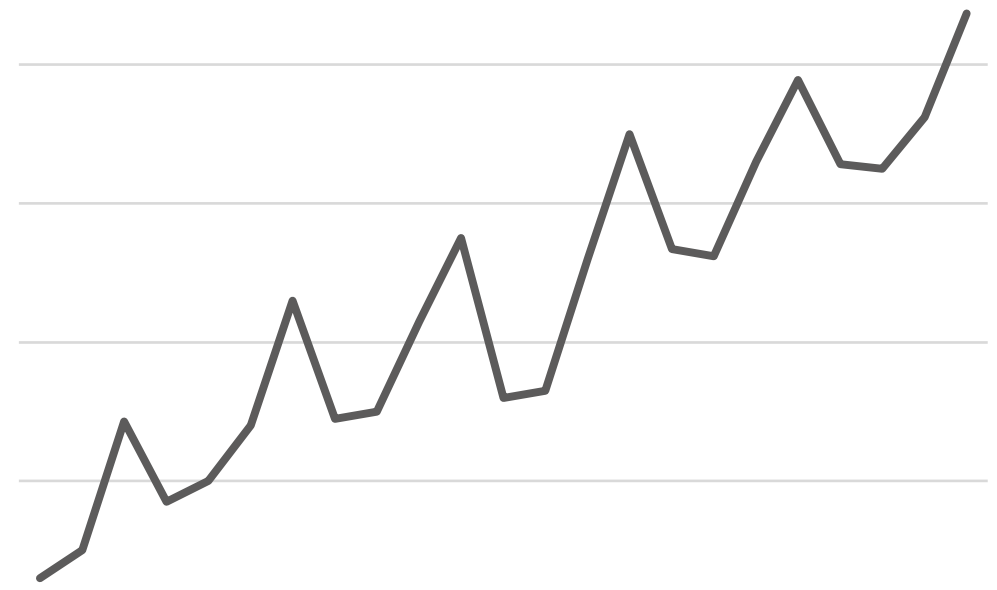

250.000

$\begin{array}{llllll}2010 & 2011 & 2012 & 2013 & 2014\end{array}$

$\mathrm{N}^{\circ}$ de deudores

Fuente: elaboración propia a partir de los microdatos de la encuesta CASEN (2013) y de la cuenta pública del año 2017 de la comisión Ingresa (2017).

Tabla 2. Discursos comunes y voces del estudiantado en relación con la categoría «Favoreciendo el acceso a la educación superior»

\begin{tabular}{ll}
\hline Discursos & Voces \\
\hline $\begin{array}{l}\text { Estudiantes consideran que el CAE ha sido } \\
\text { una oportunidad esencial para acceder a la } \\
\text { educación superior. }\end{array}$ & $\begin{array}{l}\text { Hace unos años no hubiese pensado jamás } \\
\text { entrar a la universidad. Siempre me la imaginé } \\
\text { cara. De hecho, si no fuese por el CAE, no } \\
\text { podría estar acá [en la universidad]. (Estudian- } \\
\text { te participante 1) }\end{array}$ \\
$\begin{array}{ll}\text { Estudiantes no opinan del CAE desde un posi- } \\
\text { cionamiento ideológico. Además, la mayoría } \\
\text { ignora los efectos estructurales que ha produ- } \\
\text { cido su implementación. }\end{array}$ & $\begin{array}{l}\text { el CAE. Para mí era el CAE la única opción de } \\
\text { entrar a la uni [sic], y la aproveché. (Estudiante } \\
\text { participante 5) }\end{array}$ \\
$\begin{array}{ll}\text { Estudiantes asumen que el CAE no es una } \\
\begin{array}{l}\text { ayuda en sí misma, sino que más bien con- } \\
\text { traen una deuda millonaria a pagar en años, } \\
\text { pero que se compensa por la posibilidad de } \\
\text { movilidad social que otorga el ser profesional } \\
\text { universitario. }\end{array}\end{array}$ & $\begin{array}{l}\text { Sé que hay mucha polémica con el CAE, y sé } \\
\text { termine [los estudios], pero da igual. Al final, } \\
\text { si eres universitario tienes más opciones de } \\
\text { ganar dinero. (Estudiante participante 2) }\end{array}$ \\
\hline
\end{tabular}

Fuente: elaboración propia a partir de las voces del estudiantado entrevistado. 


\section{2. ¿Equidad en el acceso?}

Primero acceso, y luego calidad y equidad. Esta parece ser la hoja de ruta de las políticas de educación superior seguida por los diversos gobiernos chilenos de la postdictadura. Este es un planteamiento que, a su vez, bascula sobre la idea matriz de que la expansión de la matrícula, independientemente de la fórmula y los expedientes utilizados para su consecución, debería proporcionar una mayor equidad al sistema. Sin embargo, dicha equidad de acceso es un concepto que puede enfocarse no solo desde la capacidad económica de los estudiantes y sus familias, sino también desde la diferenciación de distintos posicionamientos sociales, a partir de los cuales también se verifican los diferentes tipos acceso en clave interseccional: tipo de centro educativo de egreso, género, área geográfica, etnia u otro.

En el caso chileno, tanto la política pública como la literatura especializada han tendido a invisibilizar dichas variables, lo que ha generado una perspectiva donde la equidad de acceso se circunscribe por lo general al problema del financiamiento (Casanova, 2015). Por ejemplo, tal como puede verse en la tabla 3, independientemente del aumento en el número de beneficiarios del CAE para financiar sus estudios universitarios (tabla 1), los estudiantes que egresan de escuelas privadas, pertenecientes al quintil más rico de la población, siguen teniendo un acceso preferente al sistema universitario chileno, en contraposición a lo que sucede con los egresados de educación secundaria de establecimientos públicos, pertenecientes al quintil más pobre.

Tabla 3. Acceso a las universidades que participan en el sistema nacional de admisión según tipo de escuela de procedencia en el año 2015

\begin{tabular}{lrrrc}
\hline Dependencia & Rinden & Postulan & Seleccionados & Matriculados \\
\hline Público & 86.070 & 33.831 & 24.546 & 18.906 \\
\hline $\begin{array}{l}\text { Particular subvencionado / } \\
\text { Concertado }\end{array}$ & 132.825 & 68.171 & 52.136 & 41.231 \\
\hline Privado & & & & \\
\hline Sin información & 26.293 & 22.693 & 20.319 & 18.040 \\
\hline Total & 2.103 & 893 & 619 & 470 \\
\hline
\end{tabular}

Fuente: elaboración propia a partir de los datos oficiales del Ministerio de Educación (SIES, 2017).

A su vez, es posible verificar diferencias entre la equidad de acceso financiera y la académica. La primera está centrada en quiénes han sido aceptados por sus resultados en las pruebas de selección, pero que no cuentan con los recursos necesarios para ingresar, mientras que la segunda se refiere a aquellos que no han logrado los resultados requeridos en los procesos y las pruebas de selección.

En la práctica, cuando en Chile el sistema de acceso era exclusivo y universal (en la década de los setenta y hasta comienzos de los noventa) y se medía 
Tabla 4. Base total de estudiantes según quintil económico y tipo de institución de educación superior de acceso (universidades estatales o con subsidio estatal y universidades privadas)

\begin{tabular}{ccc|cc|cc}
\hline & \multicolumn{2}{c}{1990} & \multicolumn{2}{c}{2000} & \multicolumn{2}{c}{2011} \\
\cline { 2 - 7 } Quintil & IES privadas & $\begin{array}{c}\text { Universidades } \\
\text { estatales } 0 \\
\text { con subsidio } \\
\text { estatal }\end{array}$ & IES privadas & $\begin{array}{c}\text { Universidades } \\
\text { estatales } 0 \\
\text { con subsidio } \\
\text { estatal }\end{array}$ & IES privadas & $\begin{array}{c}\text { Universidades } \\
\text { estatales o con } \\
\text { subsidio estatal }\end{array}$ \\
\hline I & 46,2 & 53,8 & 43,8 & 56,2 & 66,8 & 33,2 \\
\hline II & 53,0 & 47,0 & 51,9 & 48,2 & 70,5 & 29,5 \\
\hline III & 53,5 & 46,5 & 45,2 & 54,8 & 67,1 & 32,9 \\
IV & 52,9 & 47,1 & 53,1 & 46,9 & 70,0 & 30,0 \\
\hline V & 47,3 & 52,7 & 52,5 & 47,5 & 68,8 & 31,2 \\
\hline
\end{tabular}

Fuente: elaboración propia a partir de los datos oficiales del Ministerio de Educación (SIES, 2017).

por el puntaje obtenido mediante prueba de selectividad (PSU), la equidad de acceso tenía solo una dimensión financiera, pues la selección académica la realizaba la prueba estandarizada, excluyendo previamente a aquellos que no disponían de recursos económicos. Sin embargo, la proliferación de instituciones que no subordinaron sus políticas de acceso a la rendición de tales pruebas, tal como puede verse en la tabla 4 , ampliaron significativamente la base total de estudiantes de educación superior (sobre todo a partir de los años noventa). Esto se tradujo en nuevas exigencias de equidad basadas en la promoción y el acceso al financiamiento para grupos a los que previamente, por motivos académicos, estaba vedado el ingreso a la educación terciaria.

Se ha verificado una diferenciación en los procedimientos para el ingreso en el sistema universitario, cuestión que ha incidido en nuevas demandas de equidad (Casanova, 2015). En este contexto de expansión de las demandas por la equidad de acceso financiera y académica, tal como se aprecia en la tabla 5, el estudiantado entrevistado se posiciona en la primera de estas dimensiones, demostrando fuertes convicciones respecto a la necesidad principal, que es la del acceso, y luego, una vez resuelto lo primero, adquiere relevancia el tema de la calidad y, por consiguiente, la movilidad social.

Para el estudiantado la equidad es vista como la posibilidad de acceder al CAE para ingresar en la educación superior, y se le otorga un carácter discriminador respecto del sistema de selección PSU. Cuando el requisito principal consistía en superar el filtro académico, el CAE no tenía mayor influencia en la mejora de la equidad de acceso respecto a aquellos alumnos que mayoritariamente provenían de hogares con pocos recursos, pues la selección se realizaba previamente al ingreso a la educación superior. Sin embargo, en un contexto de pluralización de los modos de acceso al sistema universitario, el CAE 
Tabla 5. Discursos comunes y voces del estudiantado en relación con la categoría «Equidad en el acceso»

\begin{tabular}{|c|c|}
\hline Discursos & Voces \\
\hline $\begin{array}{l}\text { Los estudiantes consideran que, en Chile, el } \\
\text { acceso a la educación superior basado esen- } \\
\text { cialmente en requisitos académicos resulta } \\
\text { elitista. Generaciones de jóvenes no pudieron } \\
\text { acceder en el pasado a la universidad, justa- } \\
\text { mente por no obtener la puntuación mínima } \\
\text { para postular. }\end{array}$ & $\begin{array}{l}\text { La PSU es muy difícil, además tienes que } \\
\text { venir de un buen colegio para tener posibi- } \\
\text { lidades. Además, yo ya trabajaba mientras } \\
\text { iba al colegio, y con suerte terminé la media } \\
\text { (educación secundaria). Lo tenía imposible } \\
\text { entrar en una universidad vía PSU. (Estudiante } \\
\text { participante 11) }\end{array}$ \\
\hline $\begin{array}{l}\text { Los estudiantes consideran que, si no fuera } \\
\text { por el CAE, irían a la universidad solo las cla- } \\
\text { ses más privilegiadas. El CAE, por tanto, es } \\
\text { visto como una oportunidad para la población } \\
\text { más desfavorecida. }\end{array}$ & $\begin{array}{l}\text { En mi familia, con mis primos, vamos todos } \\
\text { a la universidad. Mis vecinos de mi edad } \\
\text { también. Te da tranquilidad saber que no hay } \\
\text { obstáculos para ir a la universidad. Ahora } \\
\text { todos podemos ir, aunque seamos pobres. } \\
\text { (Estudiante participante 13) }\end{array}$ \\
\hline
\end{tabular}

Fuente: elaboración propia a partir de las voces del estudiantado entrevistado.

efectivamente posibilita el ingreso de estudiantes pertenecientes a estratos socioeconómicos medios y bajos, hecho que contribuye a desdibujar la frontera de exclusión del sistema universitario.

\subsection{Lucro para la banca y oportunidad individual}

Con la creación y la profundización del CAE como herramienta para financiar los estudios universitarios, se privatizó y se mercantilizó aún más el sistema de educación superior chileno. Se estableció un lucrativo nicho de negocio para la banca asistido por el Estado y se implementó un dispositivo de control social sobre el presente y el futuro de muchos estudiantes a través de la deuda. Precisamente en estas instituciones es donde se concentra el 66,5\% del alumnado que accede al CAE (Páez y Kremerman, 2016) y, a la vez, donde se encuentran los mayores porcentajes de CAE comprados por el Estado ${ }^{12}$, tal como se puede apreciar en la tabla 6. Solo el grupo Laureate International ${ }^{13}$, por ejemplo, ha recibido casi 600 mil millones de pesos chilenos a través del CAE ${ }^{14}$.

En este mismo sentido, para que este negocio fuera aún más atractivo para la banca, el Estado se comprometió originalmente a subsidiar a los bancos participantes, comprando cada año el $25 \%$ de los créditos entregados con un

12. Hasta el año 2015 el Estado debió hacer frente al 48\% de los créditos entregados por los bancos (por no pago de los estudiantes) (Páez y Kremerman, 2016).

13. Consorcio universitario internacional que en Chile cuenta con cinco instituciones de educación superior: el Instituto Profesional AIEP, el Instituto Profesional Escuela Moderna de Música (EMM), la Universidad Andrés Bello (UNAB), la Universidad de las Américas (UDLA) y la Universidad Viña del Mar (UVM). Más información: <https://www.laureate. net/>.

14. Equivalentes a 650 millones de euros aproximadamente (12/03/2020). 
Tabla 6. Principales instituciones de educación superior que presentan los mayores porcentajes de CAE comprados por el Estado entre 2006 y 2015

\begin{tabular}{lc}
\hline \multicolumn{1}{c}{ Institución de educación superior } & $\begin{array}{c}\text { Porcentaje de recompra } \\
\text { de créditos }\end{array}$ \\
\hline Universidad SEK & $69,8 \%$ \\
\hline Universidad Autónoma & $67,3 \%$ \\
\hline Universidad Pedro de Valdivia & $65,2 \%$ \\
\hline Universidad de Las Américas* & $63,4 \%$ \\
\hline Universidad Iberoamericana de Ciencia y Tecnología & $62,5 \%$ \\
\hline Instituto Profesional Escuela Moderna de Música* & $62,0 \%$ \\
\hline Universidad Bernardo O'Higgins & $61,3 \%$ \\
\hline Universidad del Desarrollo & $60,7 \%$ \\
\hline Universidad Adventista de Chile & $60,2 \%$ \\
\hline Instituto Profesional CIISA & $59,9 \%$ \\
\hline
\end{tabular}

* Son parte del grupo Laureate International.

Fuente: elaboración propia a partir de la información disponible en la comisión Ingresa (2017).

sobreprecio del 6\% (Ingresa, 2017). Sin embargo, entre 2006 y 2015, el fisco compró un $48 \%$ de los créditos con un sobreprecio que llega a casi un 30\% y equivale a más de 450 mil millones de pesos chilenos ${ }^{15}$ (DIPRES, 2015). En relación con los porcentajes de recompra, los bancos Corpbanca, Itaú y Scotiabank presentan más del $50 \%$ de los créditos entregados que ya han sido adquiridos por el fisco. En cuanto al recargo destaca el BCI, el cual recibió un considerable sobreprecio de $55,5 \%$ por los créditos que el fisco le compró (DIPRES, 2015). De esta forma, mientras en 2006 el CAE representaba el $2,4 \%$ del presupuesto de la partida de educación superior, el 2015 ya superaba el 35\% (Páez y Kremerman, 2016). Si consideramos los distintos tipos de créditos otorgados por la banca para financiar la educación superior, se puede observar que mientras en marzo de 2010 se registraban en torno a 270 mil jóvenes endeudados, en septiembre de 2015 ya se llegaba a casi 700 mil (Ingresa, 2017). Además, el monto de la deuda total se había triplicado. El CAE explica el $85 \%$ de la deuda total. En pocas palabras, en Chile educarse y endeudarse pareciera ser parte de la misma ecuación.

Por otro lado, en cuanto a la matrícula de los establecimientos estatales, esta solo aumentó en 17.321 estudiantes y su participación en el total de beneficiarios del CAE se redujo a solo un $15,3 \%$, con lo que progresivamente se ha ido convirtiendo en un apéndice del sistema de educación superior (Páez y Kremerman, 2016). Tal como se aprecia en el gráfico 2, vale la pena mencionar que, desde el año 2007, sobre el 30\% del presupuesto en educación superior se ha destinado a la ejecución del CAE (CASEN, 2013).

15. Equivalentes a 487 millones de euros aproximadamente (12/03/2020). 
Gráfico 2. Presupuesto ejecutado para el CAE como porcentaje del total de la partida de educación superior

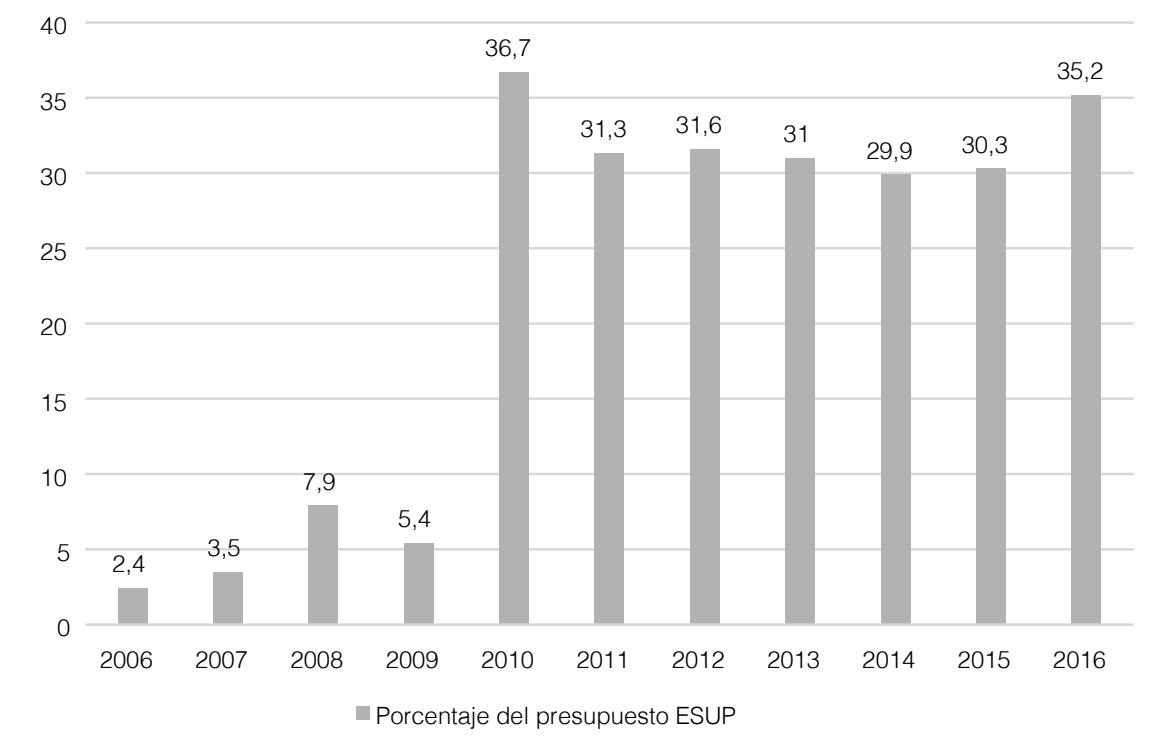

Fuente: elaboración propia a partir de los microdatos de la encuesta CASEN (2013).

$\mathrm{Al}$ respecto, tal como se aprecia en la tabla 7, el discurso de los universitarios entrevistados, aun cuando reconocen que su estatuto, en tanto estudiantes financiados con el CAE, respondía a la figura del cliente, no se soporta en un cuestionamiento a la presencia de la banca en la educación superior, sino que es legitimada — al menos de manera tácita- como una herramienta fáctica para alcanzar mayores oportunidades en la vida. En efecto, el reconocimiento subjetivo de los estudiantes en torno al CAE se da en el contexto de que el total de recursos entregados por los bancos entre 2006 y 2015 a través del CAE a las instituciones de educación superior acreditadas es de 3,44 billones de pesos chilenos ${ }^{16}$ (DIPRES, 2015) y que, más aún, solo cinco bancos se han adjudicado el 95\% de los créditos (Ingresa, 2017).

El estudiantado entrevistado no cuestiona en sí mismo el rol del Estado en tanto promotor del CAE como herramienta de financiamiento de la educación superior, ni tampoco que gran parte del financiamiento público de la educación superior (un 35\% en el año 2016) se destinara a pagar las deudas provocadas por los créditos impagados. Para el alumnado lo esencial es que una titulación universitaria le beneficiará en su calidad de vida y le ayudará a conseguir mayores ingresos monetarios, además de posicionarlo de mejor forma en el mercado laboral.

16. Equivalentes a 3.727 millones de euros aproximadamente (12/03/2020). 
Tabla 7. Discursos comunes y voces del estudiantado en relación con la categoría «Lucro para la banca y oportunidad individual»

\begin{tabular}{|c|c|}
\hline Discursos & Voces \\
\hline $\begin{array}{l}\text { El estudiantado no cuestiona el hecho de que } \\
\text { su deuda sea finalmente bancaria. Tienen } \\
\text { asumido que viven en un sistema social donde } \\
\text { la banca juega un rol esencial en todos los } \\
\text { ámbitos de la vida. Y la educación superior, por } \\
\text { tanto, sería otro ámbito más. }\end{array}$ & $\begin{array}{l}\text { Pocas cosas puedes adquirir sin préstamos } \\
\text { bancarios. Una casa, un coche, todo. Ahora yo } \\
\text { le sumo la uni [sic] y listo. Es mejor enfrentarlo } \\
\text { así. Para estudiar en la universidad debes } \\
\text { pedir un préstamo y punto. (Estudiante parti- } \\
\text { cipante 7) }\end{array}$ \\
\hline $\begin{array}{l}\text { El estudiantado manifiesta que el Estado no } \\
\text { puede financiar todo ni a todos. Más bien reco- } \\
\text { noce positivamente la gestión gubernamental } \\
\text { de poner a disposición del alumnado una } \\
\text { oportunidad de acceso a la universidad inme- } \\
\text { jorable, cuya deuda contraída le hizo ser más } \\
\text { responsable con sus estudios. }\end{array}$ & $\begin{array}{l}\text { A mí me parece bien la gestión del Estado en } \\
\text { este sentido. El hecho de saber que estudiar } \\
\text { ahora solo depende de mí me hace estar más } \\
\text { comprometida con todo el proceso: las clases, } \\
\text { las tareas, terminar la carrera pronto. (Estu- } \\
\text { diante participante 10) }\end{array}$ \\
\hline $\begin{array}{l}\text { Estudiantes no adhieren ni empatizan con las } \\
\text { reivindicaciones sociales por un cambio de } \\
\text { modelo en el financiamiento de la educación } \\
\text { superior y la supresión del CAE. }\end{array}$ & $\begin{array}{l}\text { La mayoría de los que protestan contra el CAE } \\
\text { no tienen nada que ver con nosotros. La mayo- } \\
\text { ría son acomodados y tienen todo resuelto. } \\
\text { (Estudiante participante 18) }\end{array}$ \\
\hline
\end{tabular}

Fuente: elaboración propia a partir de las voces del estudiantado entrevistado.

\section{Discusión y conclusiones}

Los principales resultados obtenidos en esta investigación demuestran que el énfasis, tanto en las políticas públicas como en los relatos de alumnos, se sitúa fundamentalmente en la problemática de la equidad de acceso a los estudios superiores. Esta focalización se debe a que en Chile ocurrió un proceso progresivo de ampliación de la base estudiantil, por lo que la problemática de la necesidad de resignificar la noción neoliberal de calidad educativa (Colella y Díaz-Salazar, 2016) quedó suspendida en el debate público, al menos hasta el desarrollo de los movimientos estudiantiles de los años 2006 y 2011.

La evidencia a la que hemos podido acceder nos muestra que los criterios de selección de los beneficiarios de la estructura del crédito con garantía estatal, focalizada en aquellos que poseen menores recursos, han sido en general exitosos en cuanto a cobertura (tabla 1). Sin embargo, aún existe un problema que apunta a las carencias del sistema educacional chileno, que no es otro que el crédito en sí mismo (gráfico 1). Las demandas expresadas en el movimiento estudiantil del año 2011 se centran en el hecho de que el CAE no es una "ayuda", sino un crédito con garantía estatal, y señalan que son los alumnos con menos recursos los que acceden en masa a esta herramienta financiera (tabla 4), de manera que adquieren una deuda que se arrastra durante años y genera claros riesgos de impago. Por tanto, el crédito con garantía estatal (CAE) se sitúa en un terreno complejo: por un lado, es considerado por el estudiantado como una herramienta vital para acceder a la educación superior (tabla 2), pero, por otro, es el inicio de una larga deuda económica, cuyo incumplimiento debe asumirlo el Estado (tabla 6). 
Otro aspecto desarrollado en el artículo permite establecer que las principales beneficiarias de la implementación del CAE son las instituciones financieras, puesto que les ha posibilitado aumentar su capacidad de mercado sin apenas riesgos (DIPRES, 2015; Ingresa, 2017).

Ahora bien, retomando la pregunta inicial planteada en la introducción del presente trabajo ( ${ }_{i}$ Cuáles son las principales coherencias e interfaces entre los resultados del CAE en tanto instrumento de acceso a la educación superior en Chile y la percepción del estudiantado beneficiario?), podemos concluir lo siguiente:

Este estudio demuestra la existencia de interfaces complejas entre la realidad educativa, la política pública y la experiencia de los estudiantes o, si se prefiere y en una perspectiva más tradicional, entre las estructuras sistémicas y las agencias de los sujetos (Sandoval y Carvallo, 2019). En efecto, mientras una mirada a la objetividad del sistema demuestra el soporte neoliberal y el refuerzo que supone la «bancarización» del financiamiento educativo — que, en la práctica, se traduce en una deuda de arrastre de los estudiantes que se perpetúa incluso por décadas-, la perspectiva de los beneficiarios es que el CAE representa una oportunidad —en muchos casos la única- de acceder a la educación superior, oportunidad a su vez que legitima al CAE «en sí», y no por su adscripción a los principios de determinada matriz ideológicoeconómica.

El acceso ha sido el principal factor explicativo en torno al grado de equidad del sistema universitario chileno. En este sentido, los estudiantes entrevistados evalúan al CAE positivamente, puesto que sin el mismo no hubieran podido acceder a la educación universitaria, y la mayoría de ellos ignora los efectos estructurales que ha producido su implementación (tabla 2).

Por último, consideramos que la complejidad de estas interfaces interactivas debe dar curso a nuevas propuestas teóricas e investigativas capaces de engarzar coherentemente la relación entre las condiciones y las posiciones estructurales, y las disposiciones y las representaciones individuales (tabla 7).

Lo señalado hasta aquí da cuenta de una realidad universitaria compleja. Por un lado, resulta evidente, a la luz de los datos recién expuestos, que los argumentos a través de los cuales se había justificado la aprobación del CAE no eran - o, al menos, ya no son- válidos. Por otro lado, la escasez presupuestaria del Estado para financiar la educación superior resulta cuestionable si se considera el continuo flujo de dinero público que, producto del CAE, ha recibido la banca, verdadera institución favorecida con esta política gubernamental.

\section{Referencias bibliográficas}

Atria, F. (2017). La mala educación: Ideas que inspiran al movimiento estudiantil de Chile. Santiago de Chile: Catalonia.

Banco Mundial (2011). Programa de Crédito con Aval del Estado (CAE) de Chile: Análisis y evaluación. Washington DC: The World Bank Press. Recuperado de $<$ https://bit.ly/3fJDK8P>. 
Bernasconi, A. y Rojas, F. (2004). Informe sobre la educación superior en Chile, 1980 2003. Santiago de Chile: Editorial Universitaria.

Brunner, J.J. (2015). New Dynamics in Latin America. International Higher Education, 71, 20-22. <https://doi.org/10.6017/ihe.2013.71.6092>

Casanova, D. (2015). Entre el pago y el mérito: Admisión estudiantil e inclusión social en las universidades chilenas. Tesis doctoral, Latin America Studies, Institute for History, Faculty of Humanities, Leiden University. Recuperado de $<$ https://bit.ly/3hOMnkp $>$.

CASEN - Encuesta de Caracterización Socioeconómica Nacional (2013). Resultados CASEN 2013. Santiago de Chile: Ministerio de Desarrollo Social de Chile. Recuperado de <http://observatorio.ministeriodesarrollosocial.gob.cl/ encuesta-casen-2013>.

Colella, L. y Díaz Salazar, R. (2016). Análisis de la calidad educativa en el discurso neoliberal. Educar, 53(2), 447-465. <https://doi.org/10.5565/rev/educar.749>

Denzin, N. (2001). The reflexive interview and a performative social science. Qualitative Research, 1(1), 23-46. <https://doi.org/10.1177/146879410100100102>

Dirección de Presupuestos del Gobierno de Chile (DiPRES) (2015). Informe de Pasivos Contingentes. Santiago de Chile: Ministerio de Hacienda de Chile. Recuperado de <https://bit.ly/3fNQUBY>.

Dooner, C. y Mena, P. (2018). Arancel de referencia v/s arancel real: Diagnóstico e interrogantes iniciales. Calidad en la Educación, 24, 287-318. <http://dx.doi.org/10.31619/caledu.n24.280>

Fernández-Ferrer, M. y Forés Miravalles, A. (2016). Evaluación del desarrollo competencial en la educación superior: La perspectiva del profesorado universitario. Educar, 54(2), 391-410. <https://doi.org/10.5565/rev/educar.799>

Giroux, H.; Rivera-Vargas, P. y Passerón, E. (2020). Pedagogía Pandémica. Reproducción Funcional o Educación Antihegemónica. Revista Internacional de Educación para la Justicia Social, 9(3), 1-7. Recuperado de <https://revistas.uam.es/riejs/ article/view/12199>.

Holstein, J.A. y Gubrium, J.F. (1995). The Active Interview. Thousand Oaks, CA: Sage.

Ingresa: Sistema de Crédito Estudios Superiores (2017). Cuenta Pública Año 2017. Santiago de Chile: Ingresa. Recuperado de <https://bit.ly/2ZaiKBJ>.

Inzunza, J.; Assael, J.; Cornejo, R. y Redondo, J. (2019). Public education and student movements: The Chilean rebellion under a neoliberal experiment. British Journal of Sociology of Education, 40(4), 490-506. <https://doi.org/10.1080/01425692.2019.1590179>

Jiménez Peralta, M. (2019). Identidad académica: Una franquicia en construcción. Educar, 55(2), 543-560. <https://doi.org/10.5565/rev/educar.960>

Larraín, C. y Zurita, S. (2008). The new student loan system in Chile's higher education. Higher Education, 55, 683-702. <https://doi.org/10.1007/s10734-007-9083-3>

Mayol, A. y Azócar, C. (2011). Politización del malestar, movilización social y transformación ideológica: El caso «Chile 2011». Polis: Revista Latinoamericana, 10(30), 163-184. <http://dx.doi.org/10.4067/S0718-65682011000300008> 
Miranda-Hiriart, G. (2016). Paradojas de la modernización del sistema universitario chileno. Polis: Revista Latinoamericana, 15(45), 345-361. <http://dx.doi.org/10.4067/S0718-65682016000300017>

PÁez, A. y Kremerman, M. (2016). Endeudar para gobernar y mercantilizar: El caso del CAE. Santiago de Chile: Fundación Sol. Recuperado de <https://fundacionsol. cl/cl_luzit_herramientas/static/wp-content/uploads/2016/08/Estudio-CAE20163-1.pdf>.

Rivera, P. y Paredes, J. P. (2008). Por una democracia incluyente y participativa. En C. Bascuñán, G. Correa, J. Maldonado y V. Sánchez. Más acá de los sueños, más allá de lo posible: la concertación en Chile, vol. 1 (pp. 251-315). Santiago de Chile: Editorial LOM.

Rivera, R.; Climent, V.; Espinoza, A. y Rivera-Vargas, P. (2018). Financiamiento de la Educación Superior en Chile a través del Crédito con Aval del Estado (CAE). Una oportunidad para la inclusión o el aumento de la brecha social. En P. RivERAVargas; J. Muñoz-SaAvedra; R. Morales Olivares y S. Butendieck-Hijerra (ed.). Políticas Públicas para la Equidad Social (pp. 93-101). Santiago de Chile: Colección Políticas Públicas, Universidad de Santiago de Chile. Recuperado de $<$ https://goo.gl/NRS5NU>.

Rivera-Polo, F.; Rivera-Vargas, P. y Alonso-Cano, C. (2018). Una mirada territorial al sistema universitario chileno. El caso de la Universidad regional de Aysén (UAY). Estudios pedagógicos, 44(1), 427-443. <https://dx.doi.org/10.4067/S0718-07052018000100427>

Rodríguez-Ponce, E. (2012). La educación superior en Chile y el rol del mercado: ¿Culpable o inocente? Ingeniare: Revista Chilena de Ingeniería, 20(1), 126-135. <http://dx.doi.org/10.4067/S0718-33052012000100013>

Salazar, J.M. y Leihy, P. (2013). El Manual Invisible: Tres décadas de políticas de educación superior en Chile (1980-2010). Archivos Analiticos de Políticas Educativas, 21(34), 1-38. <https://doi.org/10.14507/epaa.v21n34.2013>

Sandoval Moya, J. y Carvallo Gallardo, V. (2019). Una generación «sin miedo»: Análisis de discurso de jóvenes protagonistas del movimiento estudiantil chileno. Última Década, 27(51), 225-257. <http://dx.doi.org/10.4067/S0718-22362019000100225>

Scribano, A. y Sena, A. de (2009). Construcción de conocimiento en Latinoamérica: Algunas reflexiones desde la auto-etnografía como estrategia de investigación. Cinta Moebio, 34, 1-15. <https://doi.org/10.4067/S0717-554X2009000100001>

Servicio de Información de Educación Superior - SIES (2017). Informe matricula 2017 en educación superior en Chile. Santiago de Chile: Ministerio de Educación de Chile (MINEDUC). Recuperado de <https://hdl.handle.net/20.500. 12365/4613>.

Sisto, V. (2013). Entre la privatización y la reconstrucción de lo público en Chile: Movimientos estudiantiles y el debate acerca del devenir de la universidad. Horizontes Sociológicos, 1, 57-63.

Valles, M. (2007). Entrevistas cualitativas. Madrid: CIS. Cuadernos Metodológicos, 32. 